\title{
Radiotherapy of brain metastases from lung cancer: evidences and areas of research
}

\author{
Lucyna Kepka \\ Department of Radiotherapy, Military Institute of Medicine, Warsaw 04-141, Poland.
}

Correspondence to: Prof. Lucyna Kepka, MD, PhD Department of Radiotherapy, Military Institute of Medicine, Warsaw 04-141, Poland.E-mail: Ikepka@wim.mil.pl

How to cite this article: Kepka L. Radiotherapy of brain metastases from lung cancer: evidences and areas of research. $J$ Cancer Metastasis Treat2020;6:12. http://dx.doi.org/10.20517/2394-4722.2020.35

Received: 21 Apr 2020 Accepted: 21 Apr 2020 Published: 11 May 2020

Science Editor: Lucio Miele Copy Editor: Jing-Wen Zhang Production Editor: Jing Yu

Historically, the standard treatment for brain metastases (BM) from lung cancer involved neurosurgical resection and radiotherapy. Chemotherapy was applied, to a lesser extent, mainly for small-cell lung cancer (SCLC). However, with the ever-changing landscape of lung cancer therapy, the approach to the treatment of BM from lung cancer is also evolving. Generally, there has been a tendency to avoid wholebrain radiotherapy (WBRT) and to use more focused forms, i.e., stereotactic radiosurgery (SRS). Currently, in patients with WHO performance status 0-2 having up to 4 BM, local therapy (surgery or SRS) without WBRT is recommended ${ }^{[1]}$. The rationale for such an approach is based on evidence that the omission of WBRT minimizes neurocognitive toxicity and there is no difference in overall survival between local therapy (SRS or surgery) only and WBRT ${ }^{[2-4]}$. Nevertheless, a substantial proportion of lung cancer patients have multiple BM and for them, WBRT remains the primary treatment modality, unless their general performance status is very poor. For such patients with poor performance status, WBRT has no benefit over the use of steroids alone ${ }^{[5,6]}$. The prognosis of patients with BM from lung cancer is considered poor with a median overall survival of about 4 months with WBRT ${ }^{[7,8]}$. However, it was shown that WBRT in NSCLC patients with BM having EGFR mutations and ALK rearrangement had improved overall survival ${ }^{[9,10]}$. Novel therapies such as targeted agents for BM in lung cancer with driver mutations and immune checkpoint inhibitors have greater intracranial efficacy compared to conventional chemotherapy. In turn, this also promoted research towards combining these novel agents with SRS or WBRT. While the tumor's molecular status may have an impact on the decision to delay WBRT or SRS in subgroups of patients, there is insufficient data to make more definitive recommendations currently.

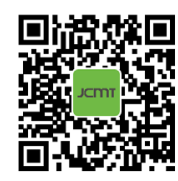


The optimal management of patients with BM from lung cancer should consider both patient (performance status, age, comorbidities, cognitive function, patient's preferences) and tumor (number/volume of BM, location, molecular subtype, extracranial disease control and systemic treatment available) related factors. There is always a trade-off between better intracranial control of disease offered by WBRT and better preservation of cognition with SRS but at the price of poorer intracranial control. This requires a detailed discussion with the patient and shared decision-making is recommended in such cases. Numerous strategies have been employed to preserve neurocognition after WBRT such as the use of neuroprotective medication and/or hippocampal avoidance (HA). Recently, the results of a randomized phase III trial comparing WBRT (30 Gy in 10 fractions)/memantine/HA to WBRT/memantine in 518 patients with multiple BM referred for WBRT were published. It was reported that WBRT with HA preserves cognitive function better after treatment compared to WBRT without HA, with no difference in intracranial control and overall survival. Thus HA should be a standard approach in all good performance status patients with BM undergoing WBRT with no metastases in the HA zone ${ }^{[11]}$.

This special issue of the Journal of Cancer Metastasis and Treatment aims to summarize the current knowledge on the treatment of BM from lung cancer with radiation by presenting the available evidence and discussing new perspectives and areas of research. The review articles by Kirakli and Yilmaz ${ }^{[12]}$ and Gutiérrez-Valencia et al. ${ }^{[13]}$ highlight the latest developments and evidence available for the use of radiotherapy in BM, and question the role of adjuvant WBRT after surgery or SRS. The issue of extending the use of SRS for more than 3-4 BM is also discussed, as we do not have evidence that directly compares such an approach to WBRT.

The planning and delivery of radiotherapy to multiple BM is a complex issue. Dumane et al ${ }^{[14]}$ present their experience with the use of knowledge-based planning, which is a new approach to treatment planning and this may lead to a fully automated planning process. Knowledge-based planning utilizes dose distributions from prior treatment plans to build a model that can predict the same for new patients. The authors then demonstrated that such an approach might be used efficiently for the complex planning involved in the use of volumetric modulated arc therapy to treat multiple BM using a single isocenter.

A comprehensive review by Mudra et al. ${ }^{[15]}$ provides the evidence regarding the use of novel systemic agents in combination with SRS for the management of BM from lung cancer. Limited evidence is available however, on the use of systemic therapy (targeted therapy, immune checkpoint inhibitors) as firstline treatment with the omission of radiation and the authors warn that such an approach should be used with caution and only for very selected patients. On the other hand, the sequence of use of systemic agents and radiation remains uncertain and is still under investigation.

In SCLC, about $40 \%-50 \%$ of patients will develop BM during the course of their disease. Owing to the distinct clinical characteristics of BM from SCLC, these patients are usually excluded from prospective trials on the value of WBRT and local ablative treatments such as surgery or SRS. Instead, WBRT in combination with chemotherapy has long been a standard approach in the setting of SCLC. However, data on the neurocognitive toxicity of WBRT, occurrence of BM after earlier use of prophylactic cranial irradiation, as well as the increasing availability of stereotactic radiotherapy technologies, have all led to the increasing use of SRS and omission of WBRT in SCLC. Various issues arising from the use of radiation for BM from SCLC in numerous clinical scenarios are discussed ${ }^{[16]}$.

Finally, this issue also contains a systematic review on the clinical effectiveness of neuroprotective agents given during WBRT: memantine, methylphenidate and donepezil. Some evidence to support the use of memantine to delay cognitive decline in patients undergoing brain irradiation was demonstrated, although at 24 weeks this did not reach statistical significance $(P=0.059)$. Despite fairly large usage of memantine 
during WBRT, more data is needed to confirm whether there is truly a long-standing neuroprotective effect of this drug for patients undergoing WBRT. Results for the two other agents were inconclusive ${ }^{[17]}$.

The Guest Editor and Contributors for this special issue of Journal of Cancer Metastasis and Treatment hope that clinicians will find these articles interesting and useful for their daily practice.

\section{DECLARATIONS}

\section{Authors' contributions}

The author contributed solely to the article.

\section{Availability of data and materials}

Not applicable.

\section{Financial support and sponsorship}

None.

\section{Conflicts of interest}

The author declared that there are no conflicts of interest.

\section{Ethical approval and consent to participate}

Not applicable.

\section{Consent for publication}

Not applicable.

\section{Copyright}

(c) The Author(s) 2020.

\section{REFERENCES}

1. Gaspar LE, Prabhu RS, Hdeib A, McCracken DJ, Lasker GF, et al. Congress of neurologic surgeons systematic review and evidencebased guideline hihges on the role of whole brain radiation therapy in adults with newly diagnosed metastatic brain tumors. Neurosurg 2019;84:e159-62.

2. Kocher M, Soffietti R, Abacioglu U, Villà S, Fauchon F, et al. Adjuvant whole-brain radiotherapy versus observation after radiosurgery or surgical resection of one to three cerebral metastases: results of the EORTC 22952-26001 study. J Clin Oncol 2011;29:134-41.

3. Brown PD, Jaeckle K, Ballman KV, Farace E, Cerhan JH, et al. Effects of radiosurgery alone vs radiosurgery with whole brain radiation therapy on cognitive function in patients with 1 to 3 brain metastases: a randomized clinical trial. JAMA 2016;316:401-9.

4. Brown PD, Ballman KV, Cerhan JH, Anderson SK, Carrero XW, et al. Postoperative stereotactic radiosurgery compared with whole brain radiotherapy for resected metastatic brain disease (NCCTG N107C/CEC.3): a multicentre, randomised, controlled, phase 3 trial. Lancet Oncol 2017; 18:1049-60.

5. Komosinska K, Kepka L, Niwinska A, Pietrzak L, Wierzchowski M, et al. Prospective evaluation of the palliative effect of whole-brain radiotherapy in patients with brain metastases and poor performance status. Acta Oncol 2010;49:382-8.

6. Mulvenna P, Nankivell M, Barton R, Faivre-Finn C, Wilson P, et al. Dexamethasone and supportive care with or without whole brain radiotherapy in treating patients with non-small cell lung cancer with brain metastases unsuitable for resection or stereotactic radiotherapy (QUARTZ): results from a phase 3, non-inferiority, randomised trial. Lancet 2016;388:2004-14.

7. Gaspar L, Scott C, Rotman M, Asbell S, Phillips T, et al. Recursive partitioning analysis (RPA) of prognostic factors in three Radiation Therapy Oncology Group (RTOG) brain metastases trials. Int J Radiat Oncol Biol Phys 1997;37:745-51.

8. Kepka L, Cieslak E, Bujko K, Wierzchowski M. Results of the whole-brain radiotherapy for patients with brain metastases from lung cancer: the RTOG RPA intra-classes analysis. Acta Oncol 2005;44:389-98.

9. Sperduto PW, Kased N, Roberge D, Xu Z, Shanley R, et al. Summary report on the graded prognostic assessment: an accurate and facile diagnosis-specific tool to estimate survival for patients with brain metastases. J Clin Oncol 2012;30:419-25.

10. Sperduto PW, Yang TJ, Beal K, Pan H, Brown PD, et al. The effect of gene alterations and tyrosine kinase inhibition on survival and cause of death in patients with adenocarcinoma of the lung and brain metastases. Int J Radiat Oncol Biol Phys 2016;96:406-13.

11. Brown PD, Gondi V, Pugh S, Tome WA, Wefel JS, et al. Hippocampal avoidance during whole-brain radiotherapy for patients with brain 
metastases: phase III trial NRG Oncology CC001. J Clin Oncol 2020;38:1019-29.

12. Kirakli EK, Yilmaz U. Radiotherapy of brain metastases from non-small cell lung cancer. J Cancer Metastasis Treat 2019;5:10.

13. Gutiérrez-Valencia E, Sánchez-Rodríguez I, Balderrama-Ibarra R, Fuentes-Lara J, Rios-Martínez A, et al. Diagnosis and management of brain metastases: an updated review from a radiation oncology perspective. J Cancer Metastasis Treat 2019;5:54.

14. Dumane VA, Tseng TC, Sheu RD, Lo YC, Gupta V, et al. Training and evaluation of a knowledge-based model for automated treatment planning of multiple brain metastases. J Cancer Metastasis Treat 2019;5:42.

15. Mudra S, Bhandari S, Tripathi P, Dunlap N, Kloecker G. Stereotactic radiosurgery in the era of novel systemic therapy for lung cancer brain metastases. J Cancer Metastasis Treat 2019;5:27.

16. Kepka L. Radiotherapy of brain metastases from small-cell lung cancer: standards and controversies. J Cancer Metastasis Treat 2019;5:53.

17. Wartena R, Brandsma D, Belderbos J. Are memantine, methylphenidate and donepezil effective in sparing cognitive functioning after brain irradiation? J Cancer Metastasis Treat 2018;4:59. 